\title{
Low-Power Inversion Recovery MRI Preserves Brain Tissue Contrast for Patients with Parkinson Disease with Deep Brain Stimulators
}

\author{
S.N. Sarkar, E. Papavassiliou, R. Rojas, D.L. Teich, D.B. Hackney, R.A. Bhadelia, J. Stormann, and R.L. Alterman
}

\begin{abstract}
BACKGROUND AND PURPOSE: Fast spin-echo short $\tau$ inversion recovery sequences have been very useful for MR imaging-guided deep brain stimulation procedures in Parkinson disease. However, high-quality fast spin-echo imaging deposits significant heat, exceeding FDA-approved limits when patients already have undergone deep brain stimulation and need a second one or a routine brain MR imaging for neurologic indications. We have developed a STIR sequence with an ultra-low specific absorption rate that meets hardware limitations and produces adequate tissue contrast in cortical and subcortical brain tissues for deep brain stimulation recipients.
\end{abstract}

MATERIALS AND METHODS: Thirteen patients with medically refractory Parkinson disease who qualified for deep brain stimulation were imaged at 1.5T with a fast spin-echo short $\tau$ inversion recovery sequence modified to meet conditional MR imaging hardware and specific absorption rate restrictions. Tissue contrast-to-noise ratios and implant localization were objectively and subjectively compared by 2 neuroradiologists, and image quality for surgical planning was assessed by a neurosurgeon for high and low specific absorption rate images.

RESULTS: The mean contrast-to-noise ratio for cerebral tissues without including the contrast-to-noise ratio for ventricular fluid was 35 and 31 for high and low specific absorption rate images. Subjective ratings for low specific absorption rate tissue contrast in $77 \%$ of patients were identical to (and in a few cases higher than) those of high specific absorption rate contrast, while the neurosurgical coordinates for fusing the stereotactic atlas with low specific absorption rate MR imaging were equivalent to those of the high specific absorption rate for $69 \%$ of patients.

CONCLUSIONS: Patients with Parkinson disease who have already had a deep brain stimulation face a risk of neural injury if routine, high specific absorption rate MR imaging is performed. Our modified fast spin-echo short $\tau$ inversion recovery sequence conforms to very conservative radiofrequency safety limits, while it maintains high tissue contrast for presurgical planning, postsurgical assessment, and radiologic evaluations with greater confidence for radiofrequency safety.

ABBREVIATIONS: CNR = contrast-to-noise ratio; DBS = deep brain stimulator or stimulation; FSTIR $=$ fast spin-echo short $\tau$ inversion recovery; SAR $=$ specific absorption rate; $\mathrm{PD}=$ Parkinson disease; $\mathrm{RF}=$ radiofrequency; $\mathrm{STN}=$ subthalamic nucleus

$\mathrm{T}$ he diagnostic quality and radiofrequency $(\mathrm{RF})$ safety of MR imaging for visualizing the subthalamic nucleus (STN) and globus pallidus are not simultaneously achievable, though both are crucial for surgical accuracy and treatment efficacy of deep brain stimulation (DBS) procedures ${ }^{1-3}$ in patients with drug-refractory Parkinson disease (PD). Kitajima et $\mathrm{al}^{3}$ observed significantly better, though not perfect, mapping of the STN by using inversion recovery (fast spin-echo short $\tau$ inversion recovery [FSTIR]) sequences. Ben-Haim et $\mathrm{al}^{4}$ reported improved STN

Received September 30, 2013; accepted after revision November 13.

From the Department of Radiology (S.N.S., R.R., D.L.T., D.B.H., R.A.B., J.S.) and Division of Neurosurgery (E.P., R.L.A.), Beth Israel Deaconess Medical Center, Harvard Medical School, Boston, Massachusetts.

Please address correspondence to Subhendra N. Sarkar, PhD, Department of Radiology, Beth Israel Deaconess Medical Center, Harvard Medical School, 330 Brookline Ave, Ansin E/245, Boston, MA 02215; e-mail: ssarkar@bidmc.harvard.edu

http://dx.doi.org/10.3174/ajnr.A3896 targeting by combining FSTIR and contrast-enhanced spoiled gradient-recalled-echo acquisitions. Although not currently approved for DBS recipients, higher fields show clear delineation of the STN at 7T. ${ }^{5-7}$

The deposited RF power (specific absorption rate [SAR]) increases with field strength; and the effective sequences, including FSTIR or T2, pose significant RF heating risk, ${ }^{8}$ which has been a potential deterrent for MR imaging of DBS recipients. ${ }^{9}$ Although experiences of incident-free routine high-SAR brain MR imaging in large groups of DBS patients have been reported ${ }^{2,10}$ and sentinel events, including serious brain injury or death, are very few, ${ }^{11}$ some researchers observed ${ }^{12}$ a greater incidence of neurologic deficits and tissue edema surrounding electrodes in DBS recipients after routine MR imaging that perhaps were not caused by the surgical procedure itself. Note that local SAR near the contact points at the DBS electrode base is unknown, and because DBS belongs to a class of critical-length implants, the SAR can be an 
Table 1: High- and low-SAR FSTIR sequence parameters for presurgical planning or postsurgical MR imaging assessment of DBS recipients with Parkinson disease

\begin{tabular}{|c|c|c|c|c|c|}
\hline Pulse Sequence & $\begin{array}{c}\text { TR/TE/TI/Matrix/Scan } \\
\text { Time (min) }\end{array}$ & $\begin{array}{l}\text { Section Thickness/ } \\
\text { FOV/No. of Avg/ } \\
\text { No. of Sections }\end{array}$ & $\begin{array}{l}\text { Echo-Train/ } \\
\text { BW (kHz) }\end{array}$ & $\begin{array}{l}\text { Refocusing Pulse } \\
\text { Width (Default Value)/ } \\
\text { Refocusing Pulse Flip } \\
\text { Angles in FSTIR }\end{array}$ & $\begin{array}{l}\text { Whole-Head SAR } \\
\text { (W/kg) (Estimated) }\end{array}$ \\
\hline $\begin{array}{l}\text { High-SAR 2D Ax } \\
\text { FSTIR (PD+TIw) }\end{array}$ & $\begin{array}{l}4 \mathrm{~s} / 12 \mathrm{~ms} / 140 \mathrm{~ms} / 256 \times \\
192 / 5: 30\end{array}$ & $3 \mathrm{~mm} / 24 \mathrm{~cm} / 2 / 30$ & $8 / \pm 15.8$ & $1.6 \mathrm{~ms}(1.6 \mathrm{~ms}) / 180^{\circ}$ & 1.5 \\
\hline $\begin{array}{l}\text { Low-SAR 2D Ax } \\
\text { FSTIR (PD+Tlw) }\end{array}$ & $\begin{array}{l}10-13 \mathrm{~s} / 11-13 \mathrm{~ms} / 130 \\
\mathrm{~ms} / 256 \times 192 / 7: 10-8: 30\end{array}$ & $\begin{array}{l}3 \mathrm{~mm} / 24 \mathrm{~cm} / 1 / 24-32 \\
\text { (Interleave) }\end{array}$ & $10 / \pm 15.8$ & $\begin{array}{l}2.6 \mathrm{~ms}(1.6) / \text { first }=110^{\circ} \text {, second }= \\
100^{\circ} \text {, rest of the refocusing } \\
\text { angles }=110^{\circ}\end{array}$ & $\leq 0.1$ \\
\hline
\end{tabular}

Note:-BW indicates bandwidth; T7w, T1-weighted; Avg, average; Ax, axial.

${ }^{a}$ Magnet strength, 1.5T; RF coil: transmit-receive 1-channel head coil.

b Short Tau FSTIR produces TI-weighted for short T1 tissues (fat, white matter darker, close to null) and proton density (PD) weighting for long T1 tissues (CSF, gray matter brighter, far away from null).

order of magnitude higher. ${ }^{13}$ Concerns about local heating and the variability of SAR among MR imaging machines ${ }^{14,15}$ have led to strict MR imaging conditional labeling. ${ }^{16,17}$ This has limited the choice of MR sequences and hardware with consequent loss of image quality. Using low-refocusing flip angle ${ }^{18,19}$ high-quality brain imaging on healthy controls at a low SAR has been possible, ${ }^{20}$ though this approach cannot be directly applied to DBS recipients due to hardware restrictions. ${ }^{16,17}$ High-quality FSE imaging seems to require use of high RF power and thus is restricted to planning the first DBS only. A repeat of the high SAR sequence for high-quality FSE is not recommended for implanting a second DBS or for revising prior ones due to excessive local SAR. We propose to minimize such risks, though without completely eliminating them, by an ultra-low SAR high-resolution sequence and to test its utility for diagnostic and presurgical use.

The primary cause of heating at the implant tips with FSE sequences is due to the fast application of multiple high flip angle refocusing RF pulses. We used a high-SAR FSTIR sequence (1.5 $\mathrm{W} / \mathrm{kg}$ ) on DBS surgical candidates (with no electrodes) for presurgical planning for the first DBS and compared the tissue contrast by performing an ultra-low SAR MR imaging $(\leq 0.1 \mathrm{~W} / \mathrm{kg}$ or 15 times lower) on the same patients for planning additional DBS or for revising the prior ones. The resulting images were assessed both subjectively and objectively for cerebral tissue contrast.

\section{MATERIALS AND METHODS}

We followed institutional ethics and research review committee guidelines for modifications of the FSTIR sequence, with RF coil and RF power restrictions following conditional DBS MR imaging guidelines. The routine high-SAR and the low-SAR versions of the FSTIR sequence were applied to DBS candidates in 2 different sessions, and tissue contrasts were compared by 3 readers.

\section{Patient Selection and MR Imaging Scan Design}

A group of 13 patients with medically refractory Parkinson disease (DBS candidates; mean disease duration, 11 years; age, 55-78 years; 5 men) were imaged (parameters in Table 1) for 2 or 3 sessions (depending on the number and type of DBS interventions) on the same day or within 7 months:

Session I: 2D high-SAR FSTIR, DBS candidates before the first DBS Session II: 2D low-SAR FSTIR on the same patients for assessing the first DBS or for presurgical coordinate planning for a second DBS
The lead localization and assessment for complications (first or second) were performed as follows: The patients were taken immediately after implantation from the operating room to the MR imaging unit, with or without the Leksell Frame (Elekta Instruments, Stockholm, Sweden) in place, to assess lead positions and to rule out intracerebral hemorrhage. The external components of the implanted lead wires were looped around the burrhole cover in the subgaleal space, creating a closed-circuit configuration. If the patient already had a pulse generator in place, vendor guidelines for safe MR imaging were followed. The head position often was slightly angled when the patient was imaged without the stereotactic frame, so the axial images were angled along the anterior/posterior commissure line to match the preoperative images.

\section{Objective Contrast-to-Noise Ratio Assessment}

Null Hypothesis. If one allows for the extra scanning time needed and also accepts a somewhat lower SNR that results from a low SAR, mean tissue contrast-to-noise ratios (CNRs) in low- $(\leq 0.1 \mathrm{~W} / \mathrm{kg}$ ) and high-SAR $(1.5 \mathrm{~W} / \mathrm{kg})$ methods are not significantly different in patients with $\mathrm{PD}$.

\section{Region-of-Interest Placement and SNR, CNR, and Statistical Significance Computation}

Eight different brain regions were bilaterally assessed for SNR computation. These were the following: temporal lobe gray matter, caudate head, body of the hippocampus, putamen, globus pallidus, thalamus, subthalamic nucleus, and ventricular fluid. Corresponding white matter ROIs were drawn in an insular region, anterior and posterior limbs of the internal capsule, substantia nigra, and corpus callosum to provide adjacent tissue signal intensities. The noise ROIs were drawn along the frequencyencoding direction to compute the CNR for the tissue pairs as

$$
\mathrm{CNR}=[\mathrm{SI}(\text { tissue } 1)-\mathrm{SI}(\text { tissue } 2)] / \mathrm{SD}(\text { air }) .
$$

A nonparametric statistical test (Wilcoxon signed rank test for dependent samples) was applied to evaluate the significance of the mean CNR differences between the high- and low-SAR images (Fig 1).

\section{Subjective Assessments}

High- and low-SAR FSTIR images were compared by 3 neuroradiologists for radiologic evaluation (D.L.T. and R.R., with $>15$ 
years of experience) and a neurosurgeon (R.L.A., with $>25$ years of experience) for surgical planning.

The scores were divided into 3 categories as follows (category fractions are reported in Tables 2 and 3):

Low-SAR images that produced higher tissue conspicuity than images from high SAR $(\mathrm{L}>\mathrm{H})$

Low-SAR images that produced lower tissue conspicuity than images from high SAR $(\mathrm{L}<\mathrm{H})$

Low-SAR images that produced tissue contrast almost equal to that of high-SAR images $(\mathrm{L}=\mathrm{H})$.

\section{RESULTS}

\section{Objective Assessments}

Tissue CNR for all 8 tissue pairs are plotted in Fig 1, indicating comparable but somewhat lower CNR for low-SAR images except for ventricular fluid. The mean CNRs of 7 tissue pairs, excluding ventricular fluid, were $26.6 \pm 10.6$ and $20.0 \pm 8.7$, while these were $35.4 \pm 27.1$ and $31 \pm 31$, after including ventricular fluid, for high and low SAR. The nonparametric Wilcoxon signed rank test revealed no significant difference in mean CNR for whole brain (W $=5, W_{\text {crit }}=3$ for $n=8$ tissue pairs at $P \leq .05$ ). However, the contrast for ventricular fluid is not useful for radiologic diagnosis or surgical planning, and with ventricular fluid excluded, the mean CNRs from high- and low-SAR results were somewhat different ( $\mathrm{W}=0, W_{\text {crit }}=2$ for $n=7$ tissue pairs at $\left.P \leq .05\right)$.

\section{Subjective Assessments}

All 3 readers concluded that FSTIR images do not differ substantially in terms of SNR or tissue contrasts between low and high SAR and that low-SAR images have adequate contrast to identify structures critical for DBS recipients (Fig 2). MR imaging-based estimations of nuclear coordinates for stereotactic planning of STN or globus pallidus targets were successful in 9/13 patients, while one or both nuclear margins less conspicuous in the remaining 4/13 patients at low SAR. The suboptimal visualization of the target (STN or globus pallidus) can force the surgeon to be more reliant on indirect targeting methods (ie, based on the anterior/posterior commissure line) and microelectrode recording to finalize the targets (Fig 3).

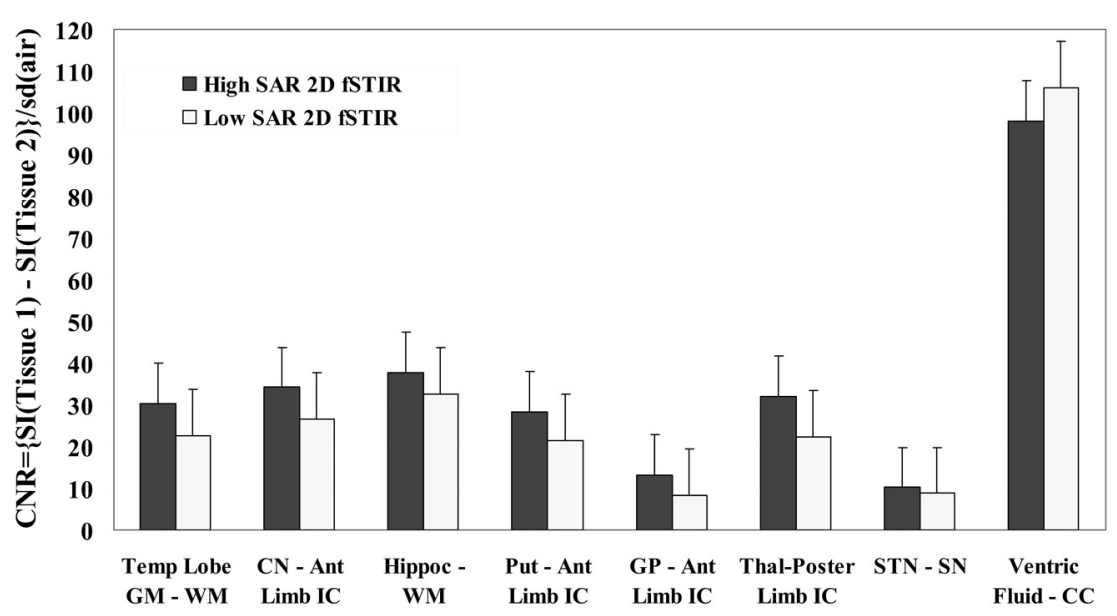

FIG 1. CNR of various tissue pairs from high- and low-SAR FSTIR sequences in 13 DBS recipients. Ant Limb or Poster Limb IC indicates anterior or posterior internal capsule; CC, corpus callosum; Put, putamen; Hippoc, body of the hippocampus; CN, caudate head; Thal, thalamus; Ventric Fluid, ventricular fluid; Temp Lobe, temporal lobe; SN, substantia nigra.

Table 2: Subjective assessment of high- and low-SAR FSTIR images of DBS candidates by various readers and percentage of patients rated for low-SAR images being higher $(L>H)$, lower $(L<H)$, or of equal utility $(L=H)$ for radiologic assessments

\begin{tabular}{lcccc}
\hline \multicolumn{1}{c}{ Readers } & $\begin{array}{c}\text { STN and RN } \\
\text { (Low vs High SAR) }\end{array}$ & $\begin{array}{c}\text { GP } \\
\text { (Low vs High SAR) }\end{array}$ & $\begin{array}{c}\text { Temporal Cortical } \\
\text { GM, CN, and Put } \\
\text { (Low vs High SAR) }\end{array}$ & $\begin{array}{c}\text { Ventricular Fluid } \\
\text { Intensity and CSF/ } \\
\text { Tissue Margins }\end{array}$ \\
\hline Neuroradiologist 1 & $\mathrm{L}>\mathrm{H}(31 \%)$ & $\mathrm{L}>\mathrm{H}(23 \%)$ & $\mathrm{L}>\mathrm{H}(23 \%)$ & $\mathrm{L}>\mathrm{H}(85 \%)$ \\
& $\mathrm{L}<\mathrm{H}(15 \%)$ & $\mathrm{L}<\mathrm{H}(15 \%)$ & $\mathrm{L}<\mathrm{H}(23 \%)$ & \\
Neuroradiologist 2 & $\mathrm{L}=\mathrm{H}(54 \%)$ & $\mathrm{L}=\mathrm{H}(62 \%)$ & $\mathrm{L}=\mathrm{H}(54 \%)$ & $\mathrm{L}=\mathrm{H}(15 \%)$ \\
& $\mathrm{L}>\mathrm{H}(23 \%)$ & $\mathrm{L}>\mathrm{H}(15 \%)$ & $\mathrm{L}>\mathrm{H}(31 \%)$ & $\mathrm{L}>\mathrm{H}(85 \%)$ \\
& $\mathrm{L}<\mathrm{H}(23 \%)$ & $\mathrm{L}=\mathrm{H}(85 \%)$ & $\mathrm{L}<\mathrm{H}(15 \%)$ & \\
& $\mathrm{L}=\mathrm{H}(54 \%)$ & $\mathrm{L}=\mathrm{H}(54 \%)$ & $\mathrm{L}=\mathrm{H}(15 \%)$ \\
\hline
\end{tabular}

Note:-L indicates low; $H$, high; RN, red nucleus; $C N$, caudate head; Put, putamen; GP, globus pallidus.

\section{DISCUSSION}

Some of the features and SAR-lowering concepts used in this work are summarized below.

High fields, in general, are associated with high SAR. The scan averages (NEX) were reduced to perform lowSAR scans within clinically feasible scanning times, which likely have contributed to a somewhat lower, though acceptable, SNR. Alternatively one could reduce the number of sections for low SAR and maintain the original number of signal averaging or use compressed sensing and a parallel imaging algorithm to further lower the SAR. The factors that lead to increased SAR and therefore should be avoided are the following: short TR, long echo trains, short RF pulses, saturation bands, driven equilibrium pulses, $100 \%$ or similarly attenuated sampling of $k$-space, and high bandwidths. Note that SAR increases quadratically with a refocusing flip angle. ${ }^{21}$ In the low-SAR version of the FSTIR sequence, we have applied the routine $180^{\circ}$ inversion and $90^{\circ}$ excitation pulses but used $<180^{\circ}$ for the train

Table 3: Subjective assessment of high- and low-SAR FSTIR images of DBS candidates by various readers and percentage of patients rated for low-SAR images being higher $(L>H)$, lower $(L<H)$, or of equal utility $(L=H)$ for surgical planning

\begin{tabular}{lcccc}
\hline & $\begin{array}{c}\text { STN/SN Contrast for } \\
\text { New DBS Plan or for } \\
\text { Prior DBS Assessment }\end{array}$ & $\begin{array}{c}\text { Putamen and GP Contrast } \\
\text { for New DBS Plan or } \\
\text { Prior DBS Assessment }\end{array}$ & $\begin{array}{c}\text { Temporal and Parietal } \\
\text { Lobe Assessment of } \\
\text { Postsurgical Complications }\end{array}$ & $\begin{array}{c}\text { Ventricular Size, Shape, and } \\
\text { Edge Detection for Planning } \\
\text { DBS Lead Trajectories }\end{array}$ \\
\hline Neurosurgeon 1 & $\mathrm{L}=\mathrm{H}(77 \%)$ & $\mathrm{L}=\mathrm{H}(69 \%)$ & $\mathrm{L}=\mathrm{H}(100 \%)$ & $\mathrm{L}=\mathrm{H}(100 \%)$ \\
\hline
\end{tabular}

Note:-L indicates low; H, high; CN, caudate head; GP, globus pallidus; SN, substantia nigra. 

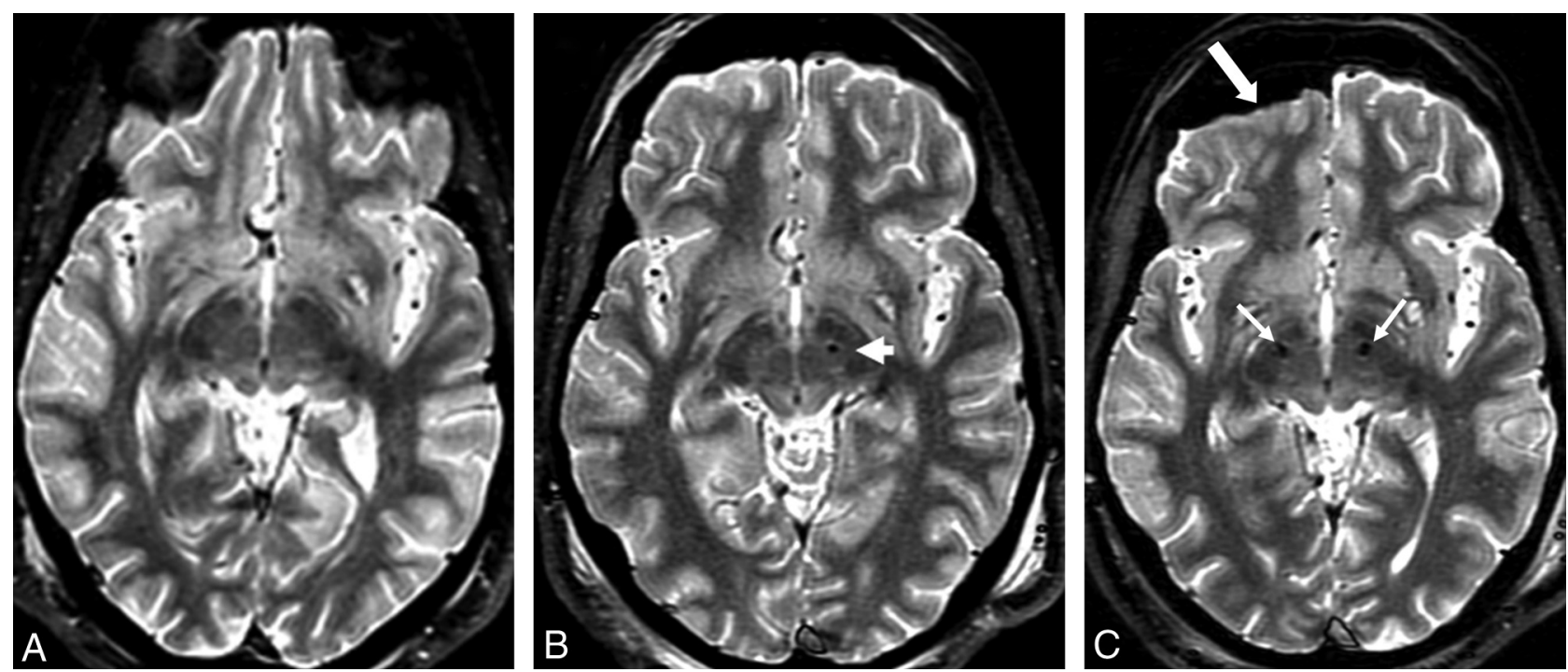

FIG 2. FSTIR 2D image sections for a typical patient scanned in 3 sessions. A, Presurgical high-SAR FSTIR image (1.5 W/kg) with interleaved 3-mm sections to plan for the first DBS implantation. B, Subsequent presurgical low-SAR FSTIR image $(0.1 \mathrm{~W} / \mathrm{kg})$ to plan for the second DBS implantation. The arrow shows the first DBS tip at the desired location of the left STN. C, Low-SAR $(0.1 \mathrm{~W} / \mathrm{kg})$ FSTIR image from the third session after a second lead implantation, to localize bilateral electrodes (arrows), visualize subcortical structures, and assess potential complications. Note the pneumocephalus (thick arrow), not uncommon during DBS implantation.
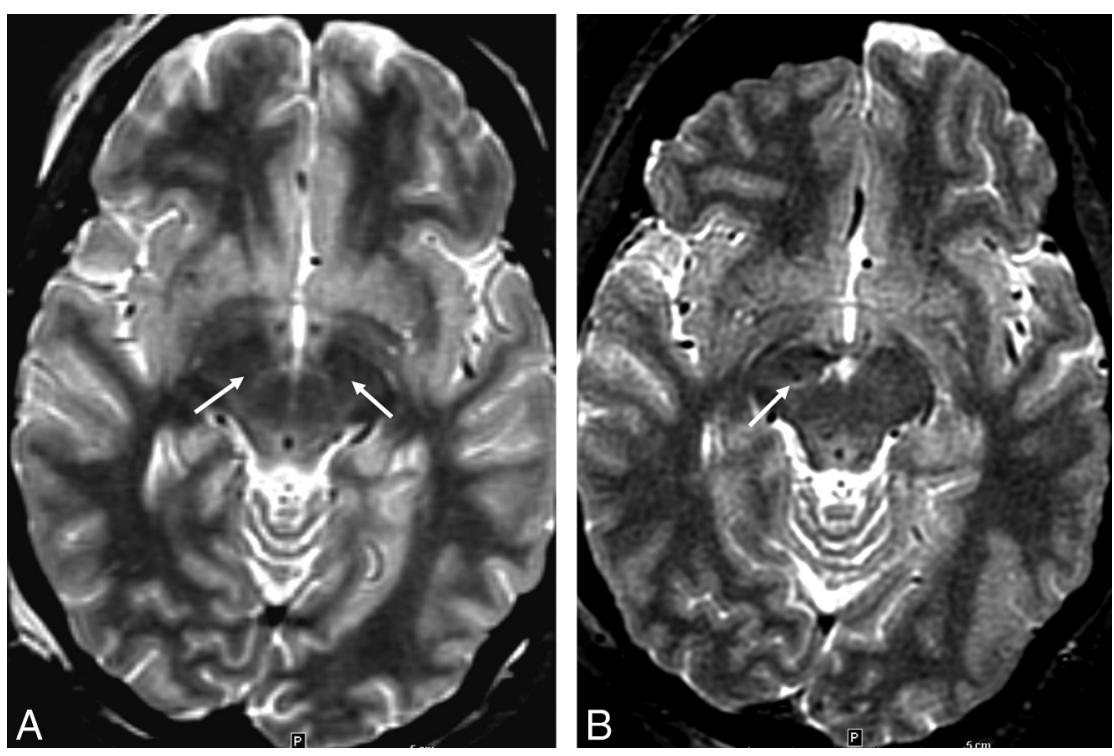

FIG 3. Example of lower STN conspicuity occasionally seen on low-SAR imaging, A, Presurgical image from high-SAR $(1.5 \mathrm{~W} / \mathrm{kg})$ FSTIR MR imaging with adequate tissue contrast allowing MR imaging-based STN coordinate measurements (arrows). B, Subsequent postsurgical low-SAR FSTIR image $(0.1 \mathrm{~W} / \mathrm{kg})$. Notice slightly lower STN contrast causing the coordinate estimation or assessment of the right lead position to be somewhat difficult (arrow) and requiring use of other landmarks and microelectrode recording.

of refocusing pulses (Table 1). This reduction in flip angle for each of the long echo trains produces a substantial reduction in energy deposition. Stretching the refocusing pulse widths (Table 1) additionally allows lower peak power and more time for heat dissipation for low echo trains. To the best of our knowledge, implementing these changes requires a research agreement. Of course, manufacturers could introduce such low-SAR sequences as product offerings.

There was a small amount of signal loss at the DBS leads in low SAR images, similar to that at high-SAR imaging reported in the literature, ${ }^{2,10}$ and far less compared with gradient-echo sequences. Gradient echo-based susceptibilityweighted imaging with phase-correction software is a low-SAR sequence and offers high conspicuity for nuclei ${ }^{22}$ and works well for preoperative MR imaging for the first DBS. However, during subsequent implantations or revisions, the metallic leads would generate significant artifacts, compromising image quality and coordinate measurements for surgical planning. Although useful, these SWIs do not reproduce the contrast characteristics of routine MR imaging, such as FSTIR.

As the use of DBS expands, there will be a greater need to use low-power MR imaging for surgical planning, evaluating implantation accuracy, and diagnosing neurologic disorders in patients with pre-existing DBS electrodes. Until fully MR imaging-compatible DBS systems are introduced and for some time after, low-SAR imaging techniques that can provide high-quality images while ensuring patient safety will be invaluable. High fields beyond $1.5 \mathrm{~T}$ offer higher SNR, but these are not yet approved for DBS patients and create challenges for MR imaging safety and RF homogeneity.

This work was performed at $1.5 \mathrm{~T}$ in full compliance with the very conservative regulatory guidelines. ${ }^{16,17}$ The high-resolution $\left(0.9 \times 1.2 \times 3 \mathrm{~mm}^{3}\right)$ low-SAR images are of diagnostic quality obtainable within clinically feasible scanning times. A moderate reduction in tissue SNR and consequently in tissue CNR at a low SAR is due to both hardware and sequence-specific limitations but provides adequate CNR for surgical planning and radiologic 
assessments. Note that the longer scanning times needed at low SARs (7-8.5 minutes versus 5.5 minutes) may increase discomfort for some patients, though in practice, we have not observed any more motion artifacts by using the low-SAR method than with high SAR, which also can lead to the patients' subjective sensation of warmth and propensity for motion. The approximate equivalence between the 2 methods suggests that the low-SAR approach can be effectively used for radiologic assessments and stereotactic targeting in the DBS patient population that is currently either being denied MR imaging or is subjected to unknown, perhaps significant tissue heating from routine, high-SAR imaging.

\section{CONCLUSIONS}

This work reports the development and radiologic quality considerations of a modified FSTIR MR imaging sequence within very conservative hardware and RF exposure constraints in the presence of implanted DBS electrodes for patients with medically refractory Parkinson disease. The low-SAR sequence seems to offer tissue contrasts for stereotactic nuclear targeting and gray/white matter structures very similar to those obtainable by using the routine high-SAR sequence and hence can be applied with greater confidence toward RF safety when additional DBSs or evaluation of existing ones is needed or potential complications are suspected. Further improvements in imaging speed and CNR may be obtained with multichannel RF coils capable of parallel imaging.

\section{ACKNOWLEDGMENTS}

The authors acknowledge the assistance of Ines Cabral-Goncalves and Raymond Airhart for administrative planning and revision of departmental policies ensuring safety for DBS patients.

Disclosures: Ron L. Alterman—UNRELATED: Consultancy: Medtronic, Comments: I receive occasional consulting fees from Medtronic regarding their DBS technology. None of that work is related to the current work. Annual compensation is less than $\$ 10,000$.

\section{REFERENCES}

1. Slavin KV, Thulborn KR, Wess C, et al. Direct visualization of the human subthalamic nucleus with 3T MR imaging. AJNR Am J Neuroradiol 2006;27:80-84

2. Larson P, Richardson R, Starr P, et al. Magnetic resonance imaging of implanted deep brain stimulators: experience in a large series. Stereotact Funct Neurosurg 2008;86:92-100

3. Kitajima M, Korogi Y, Kakeda S, et al. Human subthalamic nucleus: evaluation with high-resolution MR imaging at 3.0 T. Neuroradiology 2008;50:675-81

4. Ben-Haim S, Gologorsky Y, Monahan A, et al. Fiducial registration with spoiled gradient-echo magnetic resonance imaging enhances the accuracy of subthalamic nucleus targeting. Neurosurgery 2011;69:870-75

5. Abosch A, Yacoub E, Ugurbil K, et al. An assessment of current brain targets for deep brain stimulation surgery with susceptibilityweighted imaging at 7 Tesla. Neurosurgery 2010;67:1745-56

6. Cho Z-H, Min H-K, Oh S-H, et al. Direct visualization of deep brain stimulation targets in Parkinson disease with the use of 7-Tesla magnetic resonance imaging. J Neurosurg 2010;113:639-47

7. Eapen M, Zald DH, Gatenby JC, et al. Using high-resolution MR imaging at $7 \mathrm{~T}$ to evaluate the anatomy of the midbrain dopaminergic system. AJNR Am J Neuroradiol 2011;32:688-94

8. Finelli DA, Rezai AR, Ruggieri P, et al. MR-related heating of deep brain stimulation electrodes: an in vitro study of clinical imaging sequences. AJNR Am J Neuroradiol 2002;23:1795-802

9. Tagliati M, Jankovi J, Pagan F, et al. Safety of MRI in patients with implanted deep brain stimulation devices. Neuroimage 2009; 47(suppl 2):T53-57

10. Zrinzo L, Yoshida F, Hariz M, et al. Clinical safety of brain magnetic resonance imaging with implanted deep brain stimulation hardware: large case series and review of the literature. World Neurosurg 2011;76:164-72

11. Henderson JM, Tkach J, Phillips M, et al. Permanent neurological deficit related to magnetic resonance imaging in a patient with implanted deep brain stimulation electrodes for Parkinson's disease: case report. Neurosurgery 2005:57:E1063, discussion E1063

12. Zekaj E, Saleh C, Menghetti C, et al. Does magnetic resonance imaging induce tissue damage due to DBS lead heating? Acta Neurochir 2013;155:1677-78

13. Kainz W. MR heating tests of MR critical implants: editorial. J Magn Reson Imaging 2007;26:450-51

14. Bottomley P. Turning up the heat on MRI. J Am Coll Radiol 2008;5:853-55

15. Baker K, Tkach J, Phillips M, et al. Variability in RF-induced heating of a deep brain stimulation implant across MR systems. J Magn Reson Imaging 2006;24:1236-42

16. Medtronic. MRI Guidelines for Medtronic Deep Brain Stimulation Systems. Minneapolis: Medtronic Inc; 2007

17. Guidance for Industry and FDA Staff. Center for Devices and Radiological Health. Establishing Safety and Compatibility of Passive Implants in the Magnetic Resonance (MR) Environment. Silver Spring, Maryland; FDA; August 21, 2008; http://www.fda.gov/cdrh/osel/ guidance/1685.html

18. Busse R, Brau A, Vu A, et al. Effects of refocusing flip angle modulation and view ordering in 3D fast spin echo. Magn Reson Med 2008;60:640-49

19. Alsop DA. The sensitivity of low flip angle rare imaging. Magn Reson Med 1997;37:176-84

20. Sarkar SN, Alsop DA, Madhuranthakam AJ, et al. Brain MRI at ultralow SAR: a potential protocol for imaging of patients with contraindicating implants. Radiology 2011;259:550-57

21. Bernstein MA, King KF, Zhou XJ. Handbook of MRI Pulse Sequences. Amsterdam: Elsevier Academic Press; 2004;33

22. O'Gorman RL, Shmueli K, Ashkan K, et al. Optimal MRI methods for direct stereotactic targeting of the subthalamic nucleus and globus pallidus. Eur Radiol 2011;21:130-36 